\title{
Evidence for Significant Projectile - Target Nucleus Scattering in Single Ionization of Helium
}

\author{
M. Schulz ${ }^{1}$, R. Moshammer ${ }^{2}$, D. Fischer ${ }^{2}$, and J. Ullrich ${ }^{2}$ \\ ${ }^{1}$ University of Missouri-Rolla, Physics Department and Laboratory for Atomic, Molecular, \\ and Optical Research, Rolla, Missouri 65409, USA \\ ${ }^{2}$ Max-Planck Institut für Kernphysik, Saupfercheckweg 1, D-69117 Heidelberg, Germany
}

We present three-dimensional fully differential cross sections for single ionization of helium by ion impact at very small and very large perturbations. Clear signatures of the projectile - target nucleus interaction are identified, which manifests itself, however, through qualitatively different features in the cross sections depending on the perturbation. 
One of the fundamental processes occurring in atomic collisions is single ionization of the target atom. This process has attracted extraordinary interest because of its direct general relevance to advancing our understanding of the few-body problem [1,2]. As a result, there is a rich literature on detailed studies of single ionization [3-5]. For the case of electron impact, measurements of fully differential cross sections (FDCS) date back to the late sixties [6]. Since then, numerous studies have been performed, especially for electrons ejected into the scattering plane, i.e. the plane which is spanned by the initial and scattered projectile momentum vectors [e.g. 3,4, 7-10].

The tremendous theoretical progress in describing experimental single ionization data which was achieved in the last decade gave rise to the hope that single ionization is essentially understood. For ionization of light target atoms by high-energy electron impact, nearly perfect agreement is routinely achieved for electrons emitted into the scattering plane [e.g. 11-13]. More importantly, even at small projectile energies close to the ionization threshold, where the theoretical description of the ionization process is orders of magnitude more difficult, calculations based on various models are now in good accord with experimental data [e.g. 2, 14-16].

At large projectile energies, the FDCS for ionization by electron and ion impact are expected to converge to each other. Furthermore, the success of various theoretical models for electron impact ionization near threshold suggested that higher order effects, leading to differences between electron and ionic projectiles at small energies, are fairly well under control as well. It was therefore not necessarily expected that studies of FDCS for ion impact would provide new insight into the ionization process. A widely accepted assumption was that the largest remaining challenge was the description of ionization of 
heavy target atoms, where qualitative discrepancies between theory and experiment remain $[17,18]$

These expectations were seriously shaken by two recent developments. First, double differential cross sections (DDCS) [19] and FDCS [20] were measured for ionization of helium by highly charged ion impact. In both experiments, the perturbation (projectile charge to velocity ratio) was 4.4 , a regime which is not accessible for electron-impact ionization of helium because it would require a projectile energy below the ionization threshold. A large peak in the direction of the initial projectile momentum $\mathrm{p}_{\mathrm{o}}$ was found which was not even qualitatively reproduced by a continuum distorted wave - eikonal initial state (CDW-EIS) calculation. Second, a complete three-dimensional electron emission pattern of the FDCS was reported for $100 \mathrm{MeV} / \mathrm{amu} \mathrm{C}^{6+}+\mathrm{He}$ collisions (corresponding to a perturbation of 0.1) [1]. While in the scattering plane the data were very well reproduced by a CDW calculation, poor agreement was obtained outside the scattering plane. This was a very surprising finding as it was generally taken for granted that for such a small perturbation even the first Born approximation would provide at least an adequate qualitative description of the ionization process. As a potential explanation for these discrepancies, an incomplete treatment of the projectile - target nucleus interaction in the CDW - model was discussed.

In this letter, we provide strong experimental evidence that the features observed in the experimental data of reference [1] and not reproduced by theory can indeed be attributed to the projectile - target nucleus interaction. Furthermore, we show that the above mentioned discrepancies between experiment and theory for ionic projectiles at large and small perturbations can be traced to the same basic root. 
Two experiments were performed for $3.6 \mathrm{MeV} / \mathrm{amu} \mathrm{Au}^{53+}$ and $100 \mathrm{MeV} / \mathrm{amu} \mathrm{C}^{6+}$ projectiles. The beams collided with He-atoms from a supersonic gas jet. The projectiles which did not change charge state were selected by a switching magnet and detected by a scintillator. The recoil ions and the ionized electrons were extracted in the longitudinal direction (defined by the initial projectile direction) by a weak electric field and detected by two-dimensional position-sensitive channel plate detectors. The electron detector was set in coincidence with both the projectile and the recoil ion detectors. A uniform magnetic field of $17 \mathrm{G}\left(20 \mathrm{G}\right.$ for the $\left.\mathrm{Au}^{53+}\right)$ confined the transverse motion of the electrons so that all electrons with a transverse momentum of less than 3.0 a.u. $\left(3.5\right.$ a.u. for $\left.\mathrm{Au}^{53+}\right)$ were guided onto the detector. The transverse momenta of the electrons and the recoil ions were calculated from their position on the respective detector and their longitudinal momentum components were determined from the time of flight. The momentum vector of the scattered projectile was deduced from momentum conservation.

In Fig. 1 we show the measured (top) and calculated (bottom) three-dimensional images of the FDCS (differential in the solid angle of the ejected electron and the scattered projectile and in electron energy) for $100 \mathrm{MeV} / \mathrm{amu} \mathrm{C}^{6+}+\mathrm{He}$ reported in reference [1]. Here, the electron energy $E_{e}$ is $6.5 \mathrm{eV}$ and the momentum transfer $\mathrm{q}$ is 0.75 a.u. The calculation shows the characteristic double lobe structure familiar from electron impact studies. The large peak in the direction of $\mathbf{q}$ is called the binary peak and the smaller peak in the direction of $\mathbf{- q}$ is called the recoil peak. In the experimental data, only the binary peak is observed. However, instead of a recoil peak, we find a "recoil ring" centered at the initial projectile beam axis. As a possible explanation for this ring-shape, we offered the following two-step mechanism: in the first step the projectile kicks the electron which 
thereby gets ionized. In the second step, the projectile elastically scatters off the residual target ion, which leads to a rotation of the scattering plane. As a result, the electron is ejected out of the scattering plane leading to a filling of the pronounced minimum which sharply separates the recoil peak from the binary peak in the calculation. At a much smaller projectile energy, such a filling of the minimum was also found in a CDW-EIS calculation if the projectile - target nucleus interaction is included [21].

If this explanation is indeed correct, one might expect that effects due to the projectile target nucleus interaction show up even more sensitively in differential cross sections as a function of recoil ion and projectile parameters. The projectile - electron interaction is manifested in double differential cross sections as a function of the electron energy and the transverse component of the momentum transfer $\mathrm{q}_{\perp}$. There, a ridge of enhanced intensity (known as the Bethe ridge) extending along a line for which $\mathrm{p}_{\mathrm{e}}=\mathrm{q}_{\perp}$ is observed [19], where $\mathrm{p}_{\mathrm{e}}$ is the electron momentum. In order to study the role of the projectile - target nucleus interaction in more detail, we analyzed the equivalent cross sections, i.e. those differential in the transverse recoil ion momentum $\mathrm{p}_{\perp \perp}$ and in $\mathrm{q}_{\perp}$. These cross sections are shown in Fig. 2 for the $\mathrm{C}^{6+}$ projectiles (top) and the $\mathrm{Au}^{53+}$ projectiles (bottom) with the additional condition that the electron is emitted into the scattering plane (left) or into the plane perpendicular to the scattering plane, but including the initial projectile beam axis (right).

For the case that the electron is emitted into the scattering plane, two contributions can be identified in these cross sections. The first one is centered about a line for which $\mathrm{p}_{\mathrm{r} \perp}$ is approximately a factor of 5 smaller than $\mathrm{q}_{\perp}$. Because of momentum conservation, this implies that here the electron is emitted nearly in the direction of $\mathbf{q}$, i.e. this contribution 
can be associated with the binary peak. The second contribution is centered about a line for which $p_{\perp \perp}$ is roughly a factor of 3 larger than $q_{\perp}$. Here, the electron can be emitted in any direction relative to $\mathbf{q}$. However, an emission in the direction of $\mathbf{q}$ would require an electron momentum much larger than q, for which the cross section is small. Therefore, for the $\mathrm{C}^{6+}$ projectiles this contribution is dominated by the recoil ring. For the $\mathrm{Au}^{53+}$ projectiles, the peak in the forward direction which we reported earlier [20] may also strongly contribute.

If the electron is emitted into the perpendicular plane, $\mathrm{p}_{\mathrm{r} \perp}$ has to be larger than or equal to $\mathrm{q}_{\perp}$ because $\mathbf{q}$ is the vector sum of the electron and recoil ion momenta. Therefore, the spectra for the perpendicular plane do not contain any data in the triangle below the diagonal. Near the diagonal, we observe a clear ridge of enhanced intensity, to which we refer in the following as the "recoil ridge", similar to the Bethe ridge for the electrons. For electrons emitted into the scattering plane, the recoil ridge is completely $\left(\mathrm{C}^{6+}\right.$ projectiles) or nearly completely ( $\mathrm{Au}^{53+}$ projectiles) absent.

The data in Fig. 2 provide at least a partial confirmation of our explanation for the observation of a "recoil ring" (rather than a recoil peak) in the three-dimensional FDCS: indeed, for ionization into the perpendicular plane processes involving the projectile targetnucleus interaction are of paramount importance. However, it is not immediately clear from those data whether such processes can in fact explain the ring shape or whether they merely affect the overall magnitude of the cross sections in the perpendicular plane. In order to address this question, we analyzed the three-dimensional FDCS with the additional condition that $\mathrm{p}_{\perp}$ is not within $\mathrm{q}_{\perp} \pm 30 \%$. These cross sections are shown for the $\mathrm{C}^{6+}$ 
projectiles for the same kinematic conditions as in Fig. 1 (top) and for the $\mathrm{Au}^{53+}$ projectiles for $\mathrm{E}_{\mathrm{e}}=20 \mathrm{eV}$ and $\mathrm{q}=1.0$ a.u. (bottom) in Fig. $3 .{ }^{1}$ For comparison, we also show on the right the corresponding data without the additional anti-condition on $\mathrm{p}_{\mathrm{r} \perp}$.

From Fig. 3 it can be seen that the anti-condition on $\mathrm{p}_{\mathrm{r} \perp}$ has two pronounced effects on the three-dimensional FDCS. For the $\mathrm{C}^{6+}$ projectiles, it leads to a minimum near the perpendicular plane, separating the recoil peak (it no longer looks like a recoil ring) from the binary peak. As a result, the three-dimensional FDCS with the anti-condition resemble much more the shape one would expect from the first Born approximation. For the $\mathrm{Au}^{53+}$ projectiles, the anti-condition completely removes the peak in the direction of $\mathrm{p}_{\mathrm{o}}$ which is observed without the anti-condition. These data demonstrate that the two-step mechanism involving the projectile - target nucleus interaction is indeed responsible for the ring-like shape of the recoil peak, as we speculated earlier [1]. Furthermore, the data show that this ring-like shape observed at small perturbation and the peak in the direction of $\mathrm{p}_{\mathrm{o}}$ observed at large perturbation both can be traced to the projectile - target nucleus interaction.

At first glance, it may appear surprising that the projectile - target nucleus interaction manifests itself in very different ways depending on the perturbation. This can be understood in terms of the post-collision interaction (PCI) between the outgoing projectile and the ionized electron. The importance of this interaction at large perturbation, leading to an enhanced electron emission in the forward direction, is well established [22-24]. So far, PCI has been discussed as a second order effect in the projectile - target electron

\footnotetext{
${ }^{1}$ It should be noted that setting an additional condition on an already fully differential cross section leads to an over-determination of the kinematics, i.e. the effects due to the anti-condition in Fig. 3 are kinematically enforced. The actual new information is contained in Fig.2. and we show Fig. 3 merely to make it easier for
} 
interaction. The present data for the $\mathrm{Au}^{53+}$ show that the peak structure in the direction of $\mathrm{p}_{\mathrm{o}}$ is due to a combination of a higher-order projectile - target electron interaction and the projectile - target nucleus interaction. At small perturbation, in contrast, where PCI is known to be insignificant, the projectile - target nucleus interaction leads to a ring-like shape of the recoil peak instead of a separate peak in the direction of $p_{0}$.

It should be noted that effects due to the PCI in cross sections integrated over all projectile parameters are often well reproduced by calculations which do not include the projectile - target nucleus interaction [25]. However, the present data clearly show that such calculations right from the onset cannot correctly describe PCI effects in fully differential cross sections, like the peak in the forward direction which we observe for the $\mathrm{Au}^{53+}$ projectiles. Since here the electron momentum only has a longitudinal component, the (non-zero) transverse momentum transfer must be completely balanced by the recoil ion. Without the projectile - target nucleus interaction it may therefore be possible to correctly describe the forward emission of the ionized electrons in cross sections integrated over all q, but it is impossible to calculate $\mathrm{q}$ and therefore fully differential cross sections correctly.

It is somewhat surprising that a second order effect involving the projectile - target nucleus interaction is observable at a perturbation as small as 0.1 . The reason is that this process contributes very selectively for restricted kinematics to the ionization cross sections. For example, the anti-condition on $\mathrm{p}_{\mathrm{r} \perp}$ set on the FDCS in Fig. 3 does not affect the cross sections in the scattering plane nearly as much as in the perpendicular plane.

the reader to comprehend the relation between the recoil ridge in Fig. 2 and the features observed in the fully differential cross sections. 
Successive integration over various parameters therefore continuously reduces the relative importance of this process. Already in the doubly differential cross sections in the transverse recoil ion and projectile momenta (without any condition on the electron emission plane) effects due to the projectile - target nucleus interaction are no longer observable. Our data are therefore consistent with the general assumption that higher order effects are weak at small perturbation.

It should also be noted that the projectile - target nucleus interaction for the $\mathrm{Au}^{53+}$ projectiles is much more important relative to the $\mathrm{C}^{6+}$ projectiles than suggested by Fig. 3 . For large perturbations the peak in the forward direction increases steeply with decreasing $\mathrm{q}$ while the shape of the recoil peak at small perturbations seems to be much less sensitive to q. Furthermore, at large perturbation we do, but at small perturbation we do not observe the recoil ridge in the doubly differential cross sections without any condition on the electron emission plane.

In summary, we have extended our studies of three-dimensional fully differential cross sections for single ionization of helium by ion impact. We found clear signatures of the projectile - target nucleus interaction. We demonstrated that qualitatively different features for very small and very large perturbations observed and discussed earlier can be traced to this interaction and are thus closely related. So far, no published theoretical model has been able to reproduce these features and new approaches may be required. On the experimental side a chapter which appeared to be closed already, single ionization of helium by electron impact, should be re-opened. Measurements of three-dimensional fully differential cross sections for ionization in electron - helium collisions are important in order to study whether the features we observed are characteristic to ion - atom collisions. 
One of us (M.S.) is grateful for the hospitality of the Max Planck Institut für Kernphysik. This work was supported by NSF under grant No. PHY-0097902 and INT0224943, by GSI, the Deutsche Forschungsgemeinschaft within the Leibniz-program, the European Union, and by CIRIL (GANIL at Caen). We thank Drs. D.H. Madison, A.B. Voitkiv, T. Kirchner, and S. Jones for fruitful discussions. 


\section{Figure Captions}

Fig. 1: Three-dimensional FDCS for single ionization in $100 \mathrm{MeV} / \mathrm{amu} \mathrm{C}^{6+}+\mathrm{He}$ collisions. The electron energy is $6.5 \mathrm{eV}$ and the momentum transfer is $0.75 \mathrm{a} . \mathrm{u}$. The plot on the top shows the experimental data and the one on the bottom the calculated cross sections. The arrows labeled $\mathrm{p}_{\mathrm{o}}$ and $\mathrm{q}$ indicate the direction of the initial projectile momentum and the momentum transfer, respectively.

Fig. 2: Doubly differential cross sections (on a logarithmic scale) as a function of the transverse recoil-ion and projectile momenta for $100 \mathrm{MeV} / \mathrm{amu} \mathrm{C}^{6+}+\mathrm{He}$ (top) and 3.6 $\mathrm{MeV} / \mathrm{amu} \mathrm{Au}^{53+}+\mathrm{He}$ (bottom). In each case these cross sections are shown for the condition that the electron is emitted into the scattering plane (left) and into the plane perpendicular to q (right).

Fig. 3: Three-dimensional FDCS for single ionization of He by $100 \mathrm{MeV} / \mathrm{amu} \mathrm{C}^{6+}$ (top) and 3.6 MeV/amu $\mathrm{Au}^{53+}$ impact. The plots on the left contain the condition that the transverse recoil-ion momentum is not equal to the transverse momentum transfer within $+/-30 \%$, the plots on the right do not have that condition. 


\section{References}

[1] Schulz M., Moshammer R., Fischer D., Kollmus H., Madison D.H., Jones S., and Ullrich J., Nature $\underline{422}, 48$ (2003)

[2] Rescigno T.N., Baertschy M., Isaacs W.A., and McCurdy C.W., Science 286, 2474 (1999)

[3] Ehrhardt H., Jung K., Knoth G., and Schlemmer P., Z. Phys. D1, 3 (1986) and references therein

[4] Lahmam-Bennani A., J. Phys. B24, 2401 (1991) and references therein

[5] Rudd M.E., Kim Y.-K., Madison D.H., and Gay T.J., Rev. Mod. Phys. 64, 441 (1992) and references therein

[6] Ehrhardt H., Schulz M., Tekaat T., and Willmann K., Phys. Rev. Lett. 22, 89 (1969)

[7] Stefani G., Avaldi L., and Camilloni R., J. Phys. B23, L227 (1990)

[8] Röder J., Ehrhardt H., Bray I., Fursa D.V., McCarthy I.E., J. Phys. B29, 2103 (1996)

[9] Röder J., Ehrhardt H., Pan C., Starace A.F., Bray I., and Fursa D.V., Phys. Rev. Lett. 79, $1666(1997)$

[10] Dorn A., Elliot A, Guo X., Hurn J., Lower J., Mazevet S., McCarthy I.E., Shen Y., and Weigold E., J. Phys. B30, 4097

[11] Marchalant P., Whelan C.T., and Walters H.R.J., J. Phys. B31, 1141 (1998)

[12] Jones S. and Madison D.H., Phys. Rev. A62, 042701 (2000)

[13] M. Baertschy, T.N. Rescigno, and C.W. McCurdy, Phys. Rev. A 64, 022709 (2001)

[14] Röder J., Baertschy M., and Bray I., Phys. Rev. A67, 010702(R) (2003)

[15] Jones S., and Madison D.H., Phys. Rev. Lett. 81, 2886 (1998)

[16] Pindzola M.S. and Robicheaux F., Phys. Rev. A54, 2142 (1996) 
[17] Haynes M.A. and Lohmann B., Phys. Rev. A64, 044701 (2001)

[18] Prideaux A. and Madison D.H., Phys. Rev. A67, 052710 (2003)

[19] Moshammer R., Perumal A.N., Schulz M., Rodriguez V.D., Kollmus H., Mann R., Hagmann S., and Ullrich J., Phys. Rev. Lett 7, 223201 (2001)

[20] Schulz M., Moshammer R., Perumal A.N., and Ullrich J., J. Phys. B35, L161 (2002)

[21] Madison D.H., Foster M., Fischer D., Schulz S., Moshammer R., and Ullrich J., submitted to Phys. Rev. Lett. (2003)

[22] Crooks G.B. and Rudd M.E., Phys. Rev. Lett. 25, 1599 (1970)

[23] Schulz M., Vajnai T., Gaus A.D., Htwe W., Madison D.H., and Olson R.E., Phys. Rev. $\underline{\mathrm{A} 54}, 2951(1996)$

[24] Moshammer R., Ullrich J., Kollmus H., Schmitt W., Unverzagt M., Schmidt-Böcking H., Wood C.J., and Olson R.E., Phys. Rev. $\underline{\text { A56, }} 1351$ (1997)

[25] Moshammer R., Fainstein P.D., Schulz M., Schmitt W., Kollmus, Mann R., Hagmann S., and Ullrich J., Phys. Rev. Lett. $\underline{\text { 83, }} 4721$ (1999) 

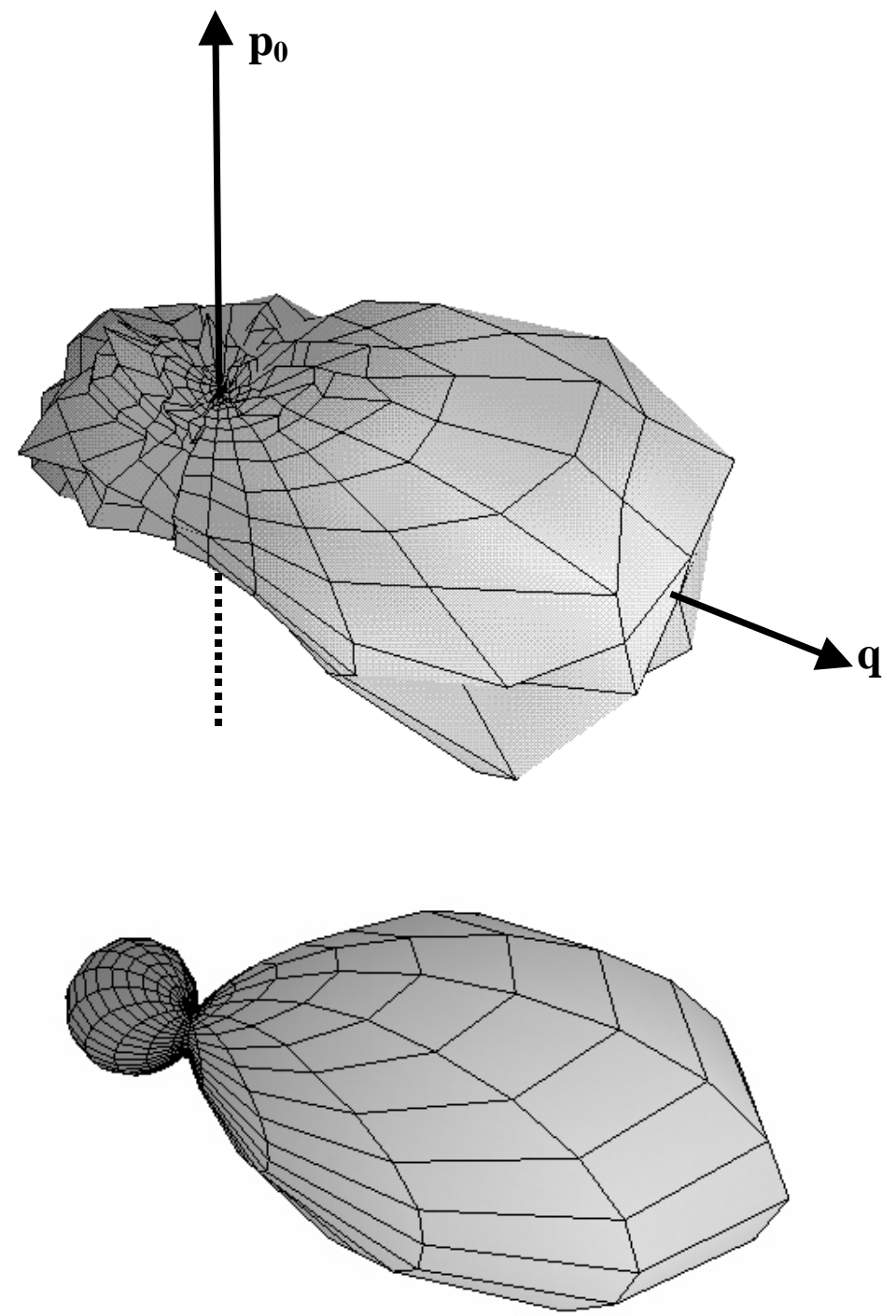

Fig. 1 

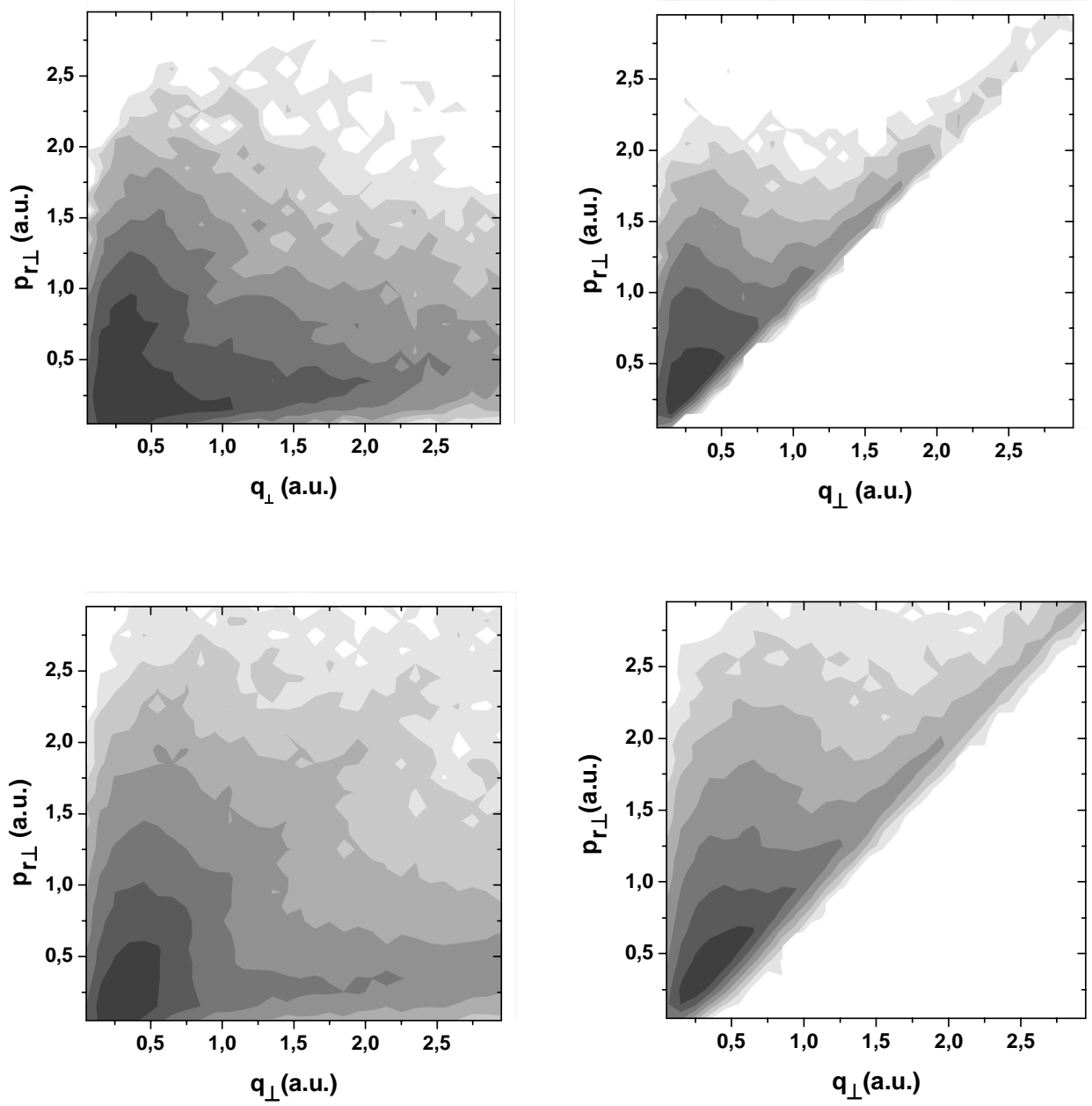

Fig. 2 

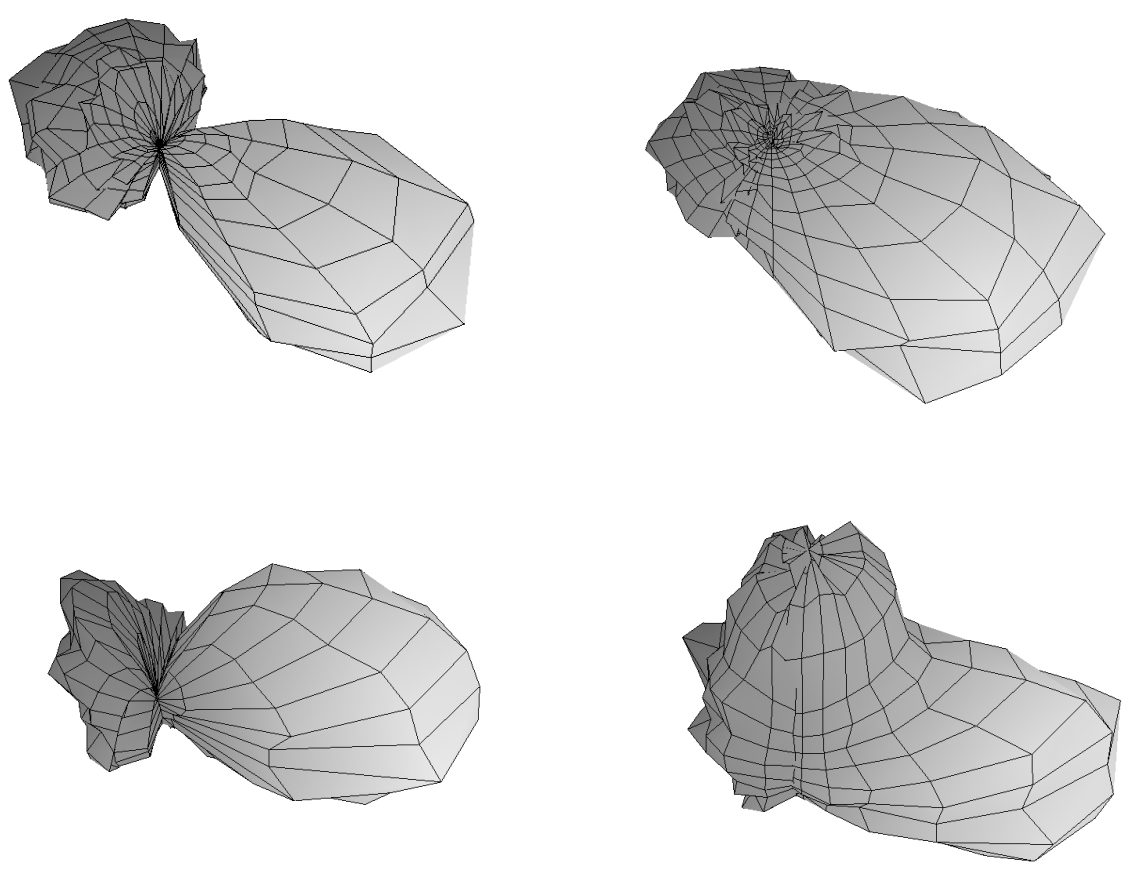

Fig. 3 